\title{
The effect of varying cone beam computed tomography image resolution and field-of-view centralization on effective radiation dose
}

Fatima M. Jadu, PhD, Asmaa A. Alzahrani, BDS, Maha A. Almutairi, BDS, Salma O. Al-Amoudi, MBBS, Ahmed M. Jan, PhD, Mawya A. Khafaji, PhD.

\begin{abstract}
الأهداف : دراسة تأثير دقة صورة الأشعة المقطعية وتوسيط المجال

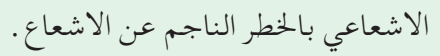

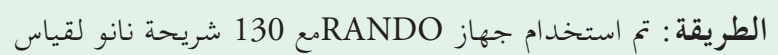

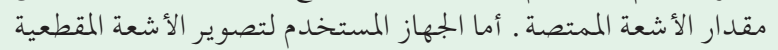

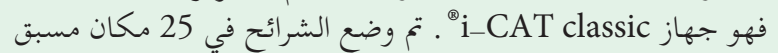

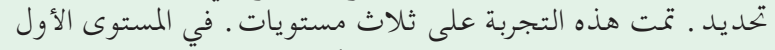

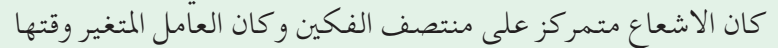

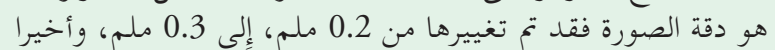

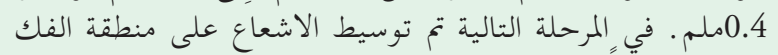

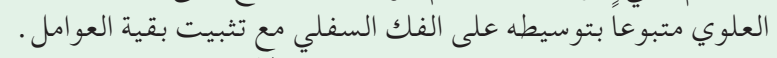

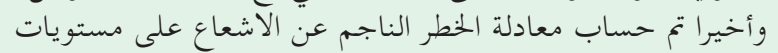
التجربة المختلفة أحسابة

النتائج: كما هو متوقع، ازدياد نسبة الخطر الناجمى عن الاشعاع عند

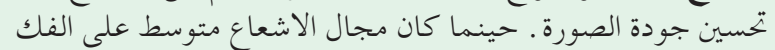

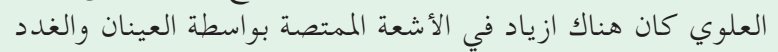

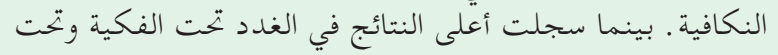

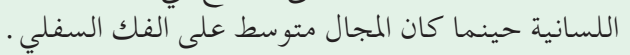

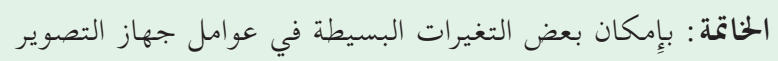

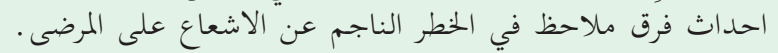

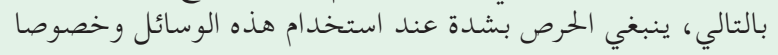

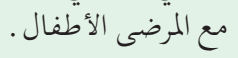

Objectives: To investigate changes in the effective radiation dose $(E)$ in relation to variations in voxel (VOX) size settings and the field-of-view (FOV) centralization.

Methods: This cross-sectional dosimetry study used nanoDot ${ }^{\mathrm{TM}}$ OSLD dosimeters placed at 25 predetermined sites in the head and neck slices of a $\mathrm{RANDO}^{\circ}$ male phantom to measure the absorbed radiation. The imaging scans took place at King Abdulaziz University Dental Hospital, Jeddah, Saudi Arabia between September 2016 and May 2017 using the i-CAT classic ${ }^{\circ}$ cone beam computed tomography (CBCT) unit. Three VOX size setting were examined: $0.2 \mathrm{~mm}, 0.3 \mathrm{~mm}$ and $0.4 \mathrm{~mm}$. The FOV was alternatively eccentrically centered on the maxillary and mandibular arches while holding all other factors constant. Effective radiation doses were calculated for each VOX size and FOV centralization setting.

Results: An inverse and indirect relation was found between $E$ and the VOX size setting with smaller VOX yielding higher $E$. CBCT scans centered on the mandible resulted in higher $E$ than scans centered on the maxilla.

Conclusion: Effective radiation doses are significantly affected by changes in VOX size settings and FOV centralization options. This reflects on the potential radiation risk to patients and highlights the importance of choosing exposure parameters carefully.

Saudi Med J 2018; Vol. 39 (5): 470-475 doi: $10.15537 /$ smj.2018.5.21658

From the Faculty of Dentistry (Jadu, Alzahrani, Almutairi, Jan), from the Department of Radiology (Khafaji), Faculty of Medicine, King Abdulaziz University; and from the Department of Radiology (Al-Amoudi), International Medical Center, Jeddah, Kingdom of Saudi Arabia.

Received 13th November 2017. Accepted 28 March 2018.

Address correspondence and reprint request to: Dr. Fatima M. Jadu, Associate Professor and Consultant, Division of Oral and Maxillofacial Radiology, Department of Oral Diagnostic Sciences, Faculty of Dentistry, King Abdulaziz University, Jeddah, Kingdom of Saudi Arabia.E-mail: fjadu@kau.edu.sa

ORCID ID: orcid.org/0000-0003-1445-3791 
A cone beam computed tomography (CBCT) becomes more popular among dentists and dental specialists, estimating the potential radiation risk to patients from this widely-used imaging technique has become crucial, especially since the use of CBCT for dental purposes involves exposing radiosensitive organs in the head and neck area such as the thyroid gland due to anatomic proximity. ${ }^{1}$ In addition, dental imaging has been hypothetically linked with an increased risk of all types of thyroid cancers and salivary gland cancers. ${ }^{2,3}$ This is especially worrisome in younger patients because the exposed organs are still developing and are at a greater risk for cancer. ${ }^{4}$

In 1975, Wolfgang Jacobi introduced the concept of effective dose in his publication "The concept of an effective dose: a proposal for the combination of organ doses" to estimate the potential risk of radiation exposure., Later, the International Commission on Radiological Protection (ICRP) incorporated the concept in their publication in 1977 as "effective dose equivalent"? In 1990, the ICRP further shortened the term into "effective dose". To date, effective dose $(E)$ is still used to estimate the potential risk of radiation exposure and its calculation depends on four factors: the measured absorbed radiation dose, the type of emitted radiation, the type and fraction of tissue irradiated. ${ }^{8}$ It is calculated by the equation:

$$
E(\mu \mathrm{Sv})=\Sigma W_{\mathrm{T}} H_{\mathrm{T}} F_{\mathrm{T}}=\Sigma W_{\mathrm{T}}\left(D_{\mathrm{T}} \mathrm{X} 1\right) F_{\mathrm{T}}
$$

Here, $W_{\mathrm{T}}$ is the weighting factor for tissue $\mathrm{T}$, the sum of all tissues $\Sigma W_{\mathrm{T}}$ is $1, H_{\mathrm{T}}$ is the equivalent radiation dose to tissue $\mathrm{T}, F_{\mathrm{T}}$ is the fraction of tissue type $\mathrm{T}$ irradiated in that view and $D_{\mathrm{T}}$ is the average absorbed radiation dose in the volume of tissue T. ${ }^{1}$ The measured absorbed radiation dose $\left(D_{\mathrm{T}}\right)$ is equal to the equivalent radiation dose $\left(H_{\mathrm{T}}\right)$ in the current study because the radiation weighting factor for $\mathrm{x}$-radiation is 1 .

In 2007, the ICRP updated their guidelines to include a change in tissue weighting factors according to new epidemiologic data on cancer incidence and mortality. ${ }^{3}$ The new guidelines included new tissues and organs such as the salivary glands, oral mucosa, and extrathoracic airway tissues, which were not considered previously. These changes resulted in an increase in $E$ calculated for all the common dental imaging techniques. The highest

Disclosure. Authors have no conflict of interests, and the work was not supported or funded by any drug company. change in $E$ was noted for bitewing radiographs made with photo-stimulable phosphor (PSP) plates and those made with $\mathrm{F}$-speed conventional film based receptors combined with rectangular collimation. ${ }^{3}$ The result was a significant change of approximately $400 \%$ in $E .^{3}$ These significant results prompted us to investigate changes in $E$ for CBCT studies based on the most recent ICRP guidelines.

The radiation dose largely depends on the technical factors used during image acquisition. The voxel (VOX) size is one such factor that can be easily modified. It controls the size of the 3 dimensional volume element. ${ }^{9}$ It thus affects the image resolution and indirectly the radiation dose. A smaller VOX size (or a greater VOX number) results in better resolution images but requires greater radiation exposure in order to avoid an increase in image noise. Image noise deteriorates the quality of the images and may render them not diagnostic. The opposite is also correct, in that larger VOX (or fewer VOX) result in less image resolution but require less radiation exposure because there is no concern for noise interfering with the quality of the images.

Another technical factor that affects the absorbed radiation dose is the field-of-view (FOV), which determines the area to be scanned and exposed to the radiation. Increasing the height or width of the FOV significantly impacts E. Different CBCT machines have different FOV sizes, but selecting a large FOV generally results in an $E$ that ranges between 46 and $916 \mu \mathrm{Sv}^{10}$ The resulting $E$ from selecting a medium FOV ranges between 47 to $560 \mu \mathrm{Sv}^{10}$ Smaller FOV results in an $E$ that ranges between 5 to $488 \mu \mathrm{Sv} .{ }^{10}$ This wide range is due to the variations in FOV centralization, which we think has a great impact on $E$. In dentistry, our interest is 'centered' on the oral cavity and the center of this region is the occlusal plane. From this perspective, maxillary and mandibular arch views are considered eccentric. Therefore, the objectives of this study were to examine the variations in $E$ from CBCT examinations when using the different available VOX size settings for the i-CAT classic ${ }^{\oplus}$ (Imaging Science International, Hatfield, PA, USA) and when alternatively varying the centralization of the FOV on the maxillary and mandibular jaws.

Methods. This dosimetry study was conducted at the Oral Radiology Department, King Abdulaziz University Dental Hospital, Jeddah, Saudi Arabia after ethical approval (IRB 041-14) was obtained. The study took place between September 2016 and May 2017. A Head and Neck RANDO ${ }^{\oplus}$ male phantom (Alderson Research Laboratories, Stanford, CT) was used for the study. 
The phantom is made of tissue-equivalent materials that mimic the radiation attenuation characteristics of human soft and hard tissues. It is sectioned horizontally into $2.5-\mathrm{cm}$ thick sections. ${ }^{11}$ Only the 10 sections representing the head and neck area of the phantom were used for this study. The absorbed radiation dose was measured at 25 preselected sites that are deemed critical radiosensitive organs or tissues in the head and neck (Table 1).

A total of 130 optically stimulated luminescent dosimeters (OSLD) (nano-Dot ${ }^{\mathrm{TM}}$, Landauer, Inc., Glenwood, IL) were used in this study. Each dosimeter (measuring $10 \times 10 \times 2 \mathrm{~mm}$ ) was used only once but was exposed to 3 cycles of radiation at a time. The absorbed radiation dose was then measured using the (MicroStar ${ }^{\circledR}$; Landauer, Inc.) reader. Unexposed nanoDot ${ }^{\mathrm{TM}}$ dosimeters were used to measure the background radiation and calibrate the other dosimeters with every set of exposures. Imaging was carried out using the i-CAT classic ${ }^{\oplus}$ (Imaging Science International, Hatfield, $\mathrm{PA}$, USA) CBCT unit. The RANDO ${ }^{\circ}$ (Alderson Research Laboratories) male phantom was oriented on the chair of the CBCT unit as demonstrated in Figure 1.

The VOX size was changed from $0.2 \mathrm{~mm}$ to 0.3 $\mathrm{mm}$ and then to $0.4 \mathrm{~mm}$ while holding all other factors constant at $16 \times 8 \mathrm{~cm}$ FOV, $10.11 \mathrm{~mA}$ and $120 \mathrm{kVp}$. This variation consequently affected the exposure time that changed from $26.9 \mathrm{sec}$ to $8.9 \mathrm{sec}$ and $4.8 \mathrm{sec}$,

Table 1 - Locations of the nanoDot ${ }^{\mathrm{TM}}$ dosimeter chips in the (RANDO) $)^{\circ}$ organs or tissue of interest.

\begin{tabular}{lc}
\hline Organ/location & Phantom level \\
\hline Brain & \\
Pituitary fossa & 4 \\
$\quad$ Sphenoid bone & 3 \\
Salivary glands & \\
$\quad$ Parotid gland (right/left) & 6 \\
$\quad$ Submandibular gland (right/left) & 8 \\
$\quad$ Sublingual gland (right/left) & 8 \\
Thyroid gland & \\
Thyroid midline & 9 \\
Thyroid surface & 9 \\
Esophagus & \\
Bone surface and marrow & 10 \\
Anterior calvarium & 2 \\
Cervical spine & 10 \\
Mandibular body (right/left) & 8 \\
Ramus (right/left) & 7 \\
Skin & \\
Lens (right/left) & 4 \\
Cheek (right/left) & 6 \\
Neck (right/left) & 8 \\
\hline
\end{tabular}

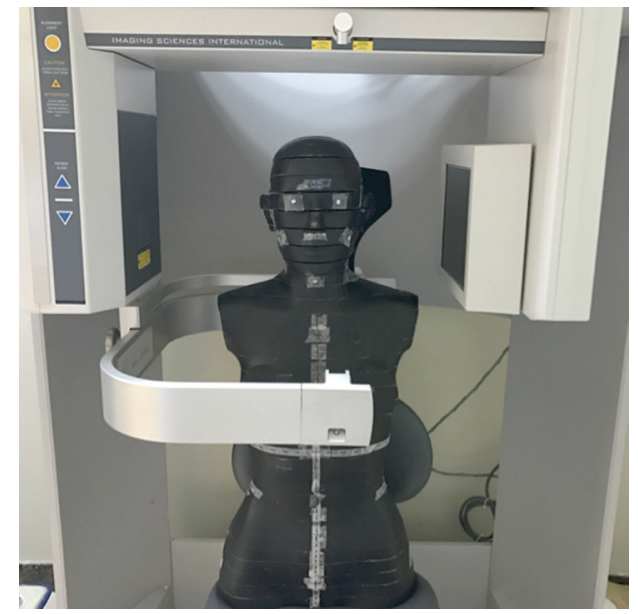

Figure 1 - The $(\text { RANDO })^{\oplus}$ male phantom in place in the i-CAT classic ${ }^{\oplus}$ cone beam computed tomography (CBCT) unit.

respectively. It also affected the image resolution. We next tested the effect of FOV centralization on $E$. All exposure factors were held constant at $8 \times 8 \mathrm{~cm}$ FOV, $10.11 \mathrm{~mA}, 120 \mathrm{kVp}$, and $0.2 \mathrm{~mm}$ VOX. The exposure time was $26.9 \mathrm{sec}$. The FOV was varied between being centered on the maxilla using the base of the nose as the guiding horizontal plane and the mandible using the mentolabial fold as the guiding horizontal plan.

Then $E$ was calculated by determining the average absorbed radiation dose in the volume of tissue and estimating the fraction of tissue irradiated in the view. We then multiplied these 2 factors by the tissueweighting factor as per the following equation:

$$
\mathrm{E}(\mu \mathrm{Sv})=\Sigma W_{\mathrm{T}} H_{\mathrm{T}} F_{\mathrm{T}}=\Sigma W_{\mathrm{T}}\left(D_{\mathrm{T}} \mathrm{X} 1\right) F_{\mathrm{T}}
$$

$W_{\mathrm{T}}$ is the tissue (T) weighting factor, the sum of all tissues $\Sigma W_{\mathrm{T}}$ is $1, H_{\mathrm{T}}$ stands for the equivalent radiation dose, $F_{\mathrm{T}}$ represents the fraction of tissue irradiated in each FOV and $D_{\mathrm{T}}$ is the average absorbed dose, is a particular organ or tissue. ${ }^{8}$ In the current study, the measured absorbed radiation dose $\left(D_{\mathrm{T}}\right)$ is equal to the equivalent radiation dose $\left(H_{\mathrm{T}}\right)$ because the radiation weighting factor for $\mathrm{x}$-radiation is 1 .

The tissue- or organ-specific weighting factors account for the variations in the risk of cancer induction or other adverse effects to that specific tissue or organ. These were based on the ICRP Publ. 103: The 2007 Recommendations of the International Commission on Radiological Protection. The fraction of tissue or organ in each FOV is estimated as demonstrated in Table 2. 
Table 2 - Estimated percentages of tissue irradiated based on the volume of that tissue in the image field.

\begin{tabular}{|c|c|c|c|}
\hline \multirow[t]{2}{*}{ Tissue } & \multicolumn{3}{|c|}{ Fraction of tissue irradiated in each FOV } \\
\hline & $\begin{array}{c}16 \times 8 \mathrm{~cm} \\
\text { centered on } \\
\text { the occlusal } \\
\text { plane }\end{array}$ & $\begin{array}{l}8 \times 8 \mathrm{~cm} \\
\text { centered on } \\
\text { the maxilla }\end{array}$ & $\begin{array}{c}8 \times 8 \mathrm{~cm} \\
\text { centered } \\
\text { on the } \\
\text { mandible }\end{array}$ \\
\hline Pituitary fossa & 10.0 & 10.0 & 5.0 \\
\hline Sphenoid & 20.0 & 20.0 & 10.0 \\
\hline Parotid gland & 100.0 & 100.0 & 60.0 \\
\hline Submandibular gland & 100.0 & 40.0 & 100.0 \\
\hline Sublingual gland & 100.0 & 40.0 & 100.0 \\
\hline Thyroid midline & 70.0 & 20.0 & 100.0 \\
\hline Thyroid surface & 70.0 & 20.0 & 100.0 \\
\hline Esophagus & 10.0 & 5.0 & 10.0 \\
\hline Bone marrow* & 3.4 & 1.9 & 2.3 \\
\hline Bone surface* & 3.6 & 2.0 & 2.6 \\
\hline $\operatorname{Skin}^{\dagger}$ & 2.5 & 1.25 & 1.25 \\
\hline Remainder tissues $^{\ddagger}$ & 100.0 & 100.0 & 100.0 \\
\hline
\end{tabular}

Values are presented as percentage.

${ }^{*}$ mandible, anterior calvarium and cervical spine, ${ }^{\dagger}$ neck, cheeks and eye lens, ${ }^{\ddagger}$ extra-thoracic tissues, and oral mucosa, FOV - field-of-view

Results. The calculated $E$ for the various CBCT scan protocols used in this study are outlined in Table 3 .

Changes in VOX size settings resulted in inverse changes in $E$ so that the greatest $E$ was calculated for the smallest VOX size setting. This result was anticipated due to the inevitable increase in exposure time that accompanies smaller VOX size settings to avoid undesirable increases in image noise. For all the VOX size settings, the highest calculated radiation dose was recorded in the remainder tissue (extrathoracic tissue and oral mucosa), followed by the bone marrow, thyroid gland and esophagus. These results are demonstrated in Figure 2.

Marked changes in $E$ were also noted with variations in FOV centralization with $E$ being greatest for CBCT scans centered on the mandible. This is likely due to the anatomic proximately of the mandible to highly radiosensitive organs such as the thyroid gland and extrathoracic tissues. Further confirmation was provided by the significant contribution of the radiation doses to these organs to the overall $E$ calculated for scans centered on the mandibular arch. On the contrary, the organs that contributed significantly to $E$ calculated for scans centered on the maxillary arch were the remainder tissue, parotid gland and bone marrow. While the organs that contributed the least lie either cranial to the orbits (such as the brain) or caudal to the mandible (such as the esophagus). These results are illustrated in Figure 3.
Table 3 - The effective radiation doses $(E)$ for the various cone beam computed tomography (CBCT) protocols explored.

\begin{tabular}{lc}
\hline CBCT scan protocol & $E(\mu \mathrm{Sv})$ \\
\hline $16 \times 8 \mathrm{~cm}$ FOV, $10.11 \mathrm{~mA}$ and $120 \mathrm{kVp}$ & 93.9 \\
$0.2 \mathrm{~mm}$ VOX, 26.9 sec & \\
centered on occlusal plane & 42.8 \\
$16 \times 8 \mathrm{~cm}$ FOV, $10.11 \mathrm{~mA}$ and $120 \mathrm{kVp}$ & \\
$0.3 \mathrm{~mm}$ VOX, $8.9 \mathrm{sec}$ & \\
centered on occlusal plane & 21.5 \\
$16 \times 8 \mathrm{~cm}$ FOV, $10.11 \mathrm{~mA}$ and $120 \mathrm{kVp}$ & \\
$0.3 \mathrm{~mm}$ VOX, $4.8 \mathrm{sec}$ \\
centered on occlusal plane \\
$8 \times 8 \mathrm{~cm}$ FOV, $10.11 \mathrm{~mA}, 120 \mathrm{kVp}$, and $0.2 \mathrm{~mm}$ VOX, \\
$26.9 \mathrm{sec}$ \\
centered on the maxillary jaw \\
$8 \times 8 \mathrm{~cm}$ FOV, $10.11 \mathrm{~mA}, 120 \mathrm{kVp}$, and $0.2 \mathrm{~mm}$ VOX, \\
$26.9 \mathrm{sec}$ \\
centered on the mandibular jaw \\
\hline \multicolumn{2}{c}{ FOV - field-of-view, VOX - voxed size } \\
\hline
\end{tabular}

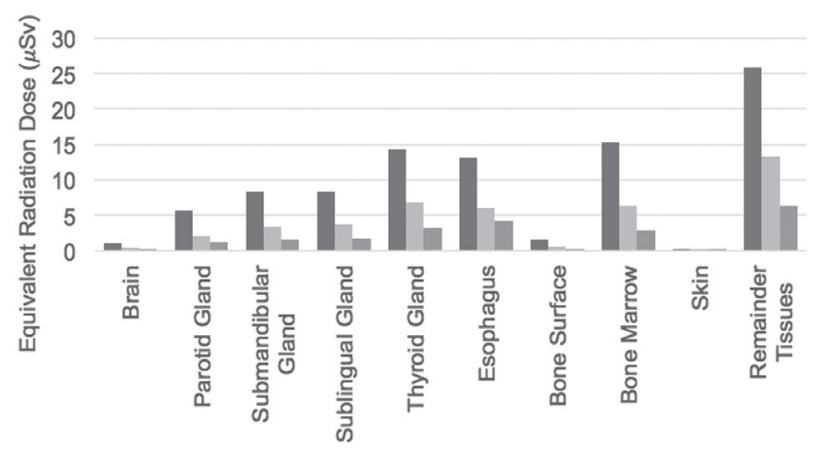

"Voxel $0.2 \mathrm{~mm}, 26.9 \mathrm{sec}$ " $=$ Voxel $0.3 \mathrm{~mm}, 8.9 \mathrm{sec}$. " Voxel $0.4 \mathrm{~mm}, 4.8 \mathrm{sec}$.

Figure 1 - Bar graph demonstrating the calculated equivalent radiation doses for the various CBCT image resolution settings.

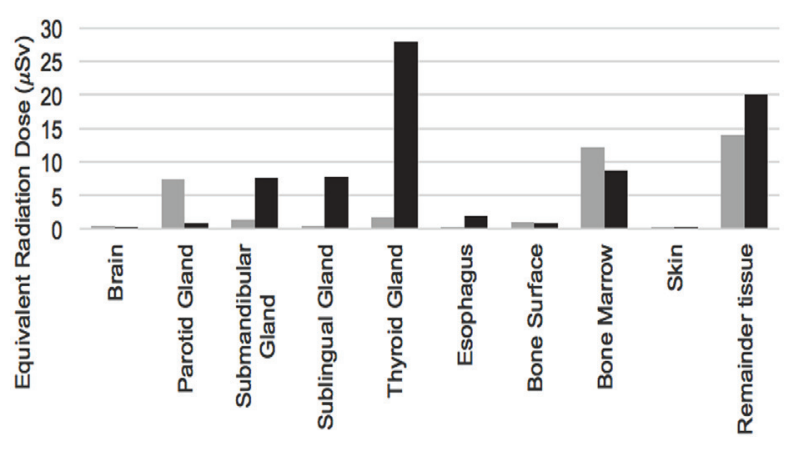

"Maxilla "Mandible

Figure 1 - Bar graph demonstrating the calculated equivalent radiation doses for CBCT scans centered on the maxilla and mandible. 
Discussion. Changes in the ICRP's guidelines have significantly changed the values of effective radiation doses for various imaging techniques and thus the estimated risk of radiation exposure to patients. These changes-combined with the gaining momentum of the "as low as diagnostically acceptable" (ALADA) 12 concept have created the need to recalculate radiation doses and re-estimate the risk of radiation exposure for common imaging procedures. This approach will enable us to gain images of diagnostic quality without overemphasizing the resolution of images in and of themselves. Additionally, practitioners must be aware of the different radiation exposure settings for each imaging modality and how varying them affects the radiation dose and the resulting image quality. This study compared different VOX size settings as well as different FOV centralization options and their impact on $E$.

As anticipated, the $E$ was inversely proportional to the VOX size settings. This is largely due to the increase in exposure time which accompanies increases in VOX settings to avoid the undesirable increase in image noise that accompanies higher resolution images. This finding agrees with several previous dosimetry studies, ${ }^{13}$ and highlights the necessity to weigh the need for high-resolution images against the added radiation risk associated with these images. Several researches have attempted to identify indications for high resolution images. ${ }^{14}$ Some found no added benefit of these images when linear measurements are required or when resorption lesions are to be assessed. ${ }^{15,16}$ Other studies showed an advantage to high resolution imaging of root fractures that are not visualized on a periapical image. ${ }^{17,18}$ Other studies demonstrated varying results when these images are used for caries and root resorption detection. ${ }^{19,20}$ We agree with the conclusion of a systemic review by Spin-Neto et al, ${ }^{14}$ that more research is needed to establish appropriate indications of high-resolution images.

Ludlow \& Ivanoc ${ }^{8}$ in a comparative dosimetry study investigated $E$ for several CBCT units using both the 1990 and the 2007 ICRP tissue weighting guidelines. They specifically explored 2 FOV sizes for the i-CAT classic $^{\circ}$ (Imaging Science International, Hatfield, PA, USA), the large setting $(16 \mathrm{~cm} \mathrm{X} 22 \mathrm{~cm})$ and the medium one $(16 \mathrm{~cm} \mathrm{X} 13 \mathrm{~cm}){ }^{8}$ The effective radiation dose for the medium FOV setting was $102 \mu \mathrm{Sv}$ and nearly doubled to $235 \mu \mathrm{Sv}$ for the large FOV setting. ${ }^{8}$ In the current study, the FOV setting was even smaller and thus yielded a smaller $E$. While they explored changes to $E$ as they relate to changes in FOV size and found a significant direct relationship, our focus was to explore changes in $E$ as they related to FOV centralization and also demonstrated significant changes. The effective radiation dose was found to be higher for CBCT scan centered on the mandibular arch primarily due to the higher doses calculated for the extrathoracic tissue and thyroid gland. Similarly, FOV centralization on the mandible has been shown to result in higher doses to the esophagus as well as the thyroid and salivary glands. ${ }^{21}$ Our research considered the parotid glands separately and demonstrated that maxillary centralization results in a markedly higher $E$ in the parotid glands. Furthermore, larger FOV settings, increase the radiation dose to the patient and may not necessarily increase the diagnostic gain ${ }^{22}$ therefore, care must be exercised when choosing this parameter in terms of size and centralization.

It is critical to maintain a balance between the diagnostic quality of an image and patient radiation exposure. Dentists should be mindful of this when requesting imaging for their patients. They should be careful in their choice of exposure parameters because this significantly affects the patient's radiation dose. Finally, the "ALADA" concept must be applied to every patient regardless of the type of imaging required. This approach, as per the NCRP's recommendation in $2014,{ }^{12}$ aims to reduce unnecessary high resolution imaging associated with greater radiation in favor of diagnostic images that may be of lower quality but associated with less radiation. ${ }^{10,23}$ Therefore, Further studies need to be carried out on the quality of the resulting images while varying the technical factors so that proper protocols can be established for the various CBCT indications that take into consideration not only the radiation risk but also the quality of the image.

In conclusion, there is unanimous agreement that variations in the CBCT exposure factors significantly affect $E$ and thus the patient's potential radiation risk. Therefore, care must be taken when choosing these parameters-especially for vulnerable patients such as children.

\section{References}

1. Jadu F, Yaffe MJ, Lam EWN. A comparative study of the effective radiation doses from cone beam computed tomography and plain radiography for sialography. Dentomaxillofacial Radiol 2010; 39: 257-263.

2. Neta G, Rajaraman P, Berrington De Gonzalez A, Doody MM, Alexander BH, Preston D, et al. A prospective study of medical diagnostic radiography and risk of thyroid cancer. Am J Epidemiol 2013; 177: 800-809. 
3. Ludlow JB, Davies-Ludlow LE, White SC. Patient risk related to common dental radiographic examinations: the impact of 2007 International Commission on Radiological Protection recommendations regarding dose calculation. J Am Dent Assoc 2008; 139: 1237-1243.

4. Gilbert ES. Ionizing Radiation and Cancer Risks: What have we learned. Int J Radiat Biol 2010; 85: 467-482.

5. Jaeobi W. The concept of the effective dose. A proposal for the combination of organ doses. Biophys 1975; 12: 1-109.

6. Streffer C, Jacob P. Wolfgang Jacobi (17 May 1928-3 March 2015). J Radiol Prot 2015; 35: 735-736.

7. International Commission on Radiological Protection. Recommendations of the International Commission on Radiological Protection. ICRP Publication 26. Ann ICRP 1977; 1: 2-3.

8. Ludlow JB, Ivanovic M. Comparative dosimetry of dental CBCT devices and 64-slice CT for oral and maxillofacial radiology. Oral Surg Oral Med Oral Pathol Oral Radiol Endod 2008; 106: 930-938.

9. Hendee WR, Ritenour ER, Hoffmann KR, editors. Medical Imaging Physics. 4th ed. New York; Wiley \& Sons Inc.; 2003. p. 730 .

10. Ludlow JB, Timothy R, Walker C, Hunter R, Benavides E, Samuelson DB, et al. Effective dose of dental CBCT - A meta analysis of published data and additional data for nine CBCT units. Dentomaxillofac Radiol 2015; 44: 20140197.

11. Radiology Support Devices. Manual. The Aldeson Radiation Theraphy Phantom [Accessed 2018 April 9]. Available from: http://www.rsdphantoms.com/pdf/aldersonradiationtherapy. pdf

12. Program AM. Annual Meeting Program NCRP: Achievements of the Past 50 Years and Addressing the Needs of the Future NCRP Mission: To support radiation protection by 2014 [Accessed 2018 April 9]. Available from: http://ncrponline.org/ wp-content/themes/ncrp/PDFs/PROGRAM_2-10.pdf

13. Roberts JA, Drage NA, Davies J, Thomas DW. Effective dose from cone beam CT examinations in dentistry. Br J Radiol 2009; 82: 35-40.
14. Spin-Neto R, Gotfredsen E, Wenzel A. Impact of voxel size variation on CBCT-based diagnostic outcome in dentistry: A systematic review. J Digit Imaging 2013; 26: 813-820.

15. Sherrard JF, Rossouw PE, Benson BW, Carrillo R, Buschang $\mathrm{PH}$. Accuracy and reliability of tooth and root lengths measured on cone-beam computed tomographs. Am J Orthod Dentofacial Orthop 2010; 137 (4 Suppl): S100-S108.

16. Nikneshan S, Valizadeh S, Javanmard A, Alibakhshi L. Effect of Voxel size on detection of external root resorption defects using cone beam computed tomography. Iran J Radiol 2016; 13: e34985.

17. Melo SLS, Bortoluzzi EA, Abreu M, Corrêa LR, Corrêa M. Diagnostic ability of a cone-beam computed tomography scan to assess longitudinal root fractures in prosthetically treated teeth. J Endod 2010; 36: 1879-1882.

18. Wenzel A, Haiter-neto F. Variable-resolution cone-beam computerized tomography with enhancement filtration compared with intraoral photostimulable phosphor radiography in detection of transverse root fractures in an in vitro model. Oral Surg Oral Med Oral Pathol Oral Radiol Endod 2009; 108: 939-945.

19. Kamburolu K, Murat S, Yüksel SP, Cebeci ARI, Paksoy CS. Occlusal caries detection by using a cone-beam CT with different voxel resolutions and a digital intraoral sensor. Oral Surg Oral Med Oral Pathol Oral Radiol Endod 2010; 109: e63-e69.

20. Safi Y, Ghaedsharaf S, Aziz A, Hosseinpour S, Mortazavi H. Effect of field of view on detection of external root resorption in cone-beam computed tomography. Iran Endod J 2017; 12: 179-184.

21. Loubele M, Bogaerts R, Van Dijck E, Pauwels R, Vanheusden S, Suetens $\mathrm{P}$, et al. Comparison between effective radiation dose of CBCT and MSCT scanners for dentomaxillofacial applications. Eur J Radiol 2009; 71: 461-468.

22. Hedeşiu M, Báciuț M, Báciuț G, Nackaerts O, Jacobs R. Comparison of cone beam CT device and field of view for the detection of simulated periapical bone lesions. Dentomaxillofacial Radiol 2012; 41: 548-552.

23. Jaju PP, Jaju SP. Cone-beam computed tomography: Time to move from ALARA to ALADA. Imaging Sci Dent 2015; 45: 263-265. 